\title{
Offloading techniques for diabetic foot
}

\begin{abstract}
Diabetes Mellitus (DM), a serious chronic disease is known to have many complications. Foot complications are one of the most distressing and affect around $15 \%$ of all diabetics. ${ }^{1}$ By 2008, around 314-382 million were people affected by DM. It has been predicted that the number of diabetics will increase by a double in 2030. With this predicted increase in prevalence of DM, the increase in the complications of DM is likely. Major causes of morbidity and increased financial burden in a diabetic patient are foot ulcers and amputations, which with early detection and management can delay or even prevent the onset of adverse outcomes. ${ }^{2}$ Hence, it is recommended that every diabetic patient should have foot evaluation at yearly intervals for risk factors of foot ulcers and amputations that include neuropathy, vasculopathy and deformities. ${ }^{3}$ Optimal management entails a multidisciplinary approach for prevention, early evaluation and treatment strategies.

The cornerstone of the management are appropriate wound care (and debridement), infection control and pressure reduction. The pathogenesis of neuropathic diabetic foot ulcers involves mechanical trauma due to focal pressure and repetitive moderate stress. ${ }^{4}$ This pressure causes maximum damage at the edge of the area of pressure application and has been termed as "edge effect". ${ }^{5}$ Triad of peripheral neuropathy, foot deformity and minor trauma have been found as risk factors in majority of cases of diabetic foot ulcers. ${ }^{6}$ Offloading the affected foot has been used as a useful management strategy as it allows for the pressure to be spread over a wider area away from the affected part. A variety of offloading techniques are in use including surgical offloading. The aim of the article is to present the various novel offloading techniques at present and their pros and cons.
\end{abstract}

Keywords: diabetes mellitus, diabetic foot, offloading, plantar ulcers, pedar ${ }^{\circledR}$, glycaemic, moderate, vasculopathy, TCC, crow
Volume 4 Issue 3 - 2017

\author{
Simerjit Singh, Ming Yoong, Avneet Kaur \\ Department of Orthopaedics, Asia
}

Correspondence: Simerjit Singh, Department of Orthopaedics, Melaka Manipal Medical College, Asia, Emailsimer1980@yahoo.com

Received:October 29, 2016 | Published: April 21, 2017
Abbreviations: DM, diabetes mellitus; TCC, total contact cast; UKPDS, uk prospective diabetes study; RCWs, removable castwalkers; CROW, charcot restraint orthotic walker; CAM, controlled ankle motion; iTCC, instant total contact cast

\section{Introduction}

\section{Pressure forces on the foot}

The sole of the foot does not have equal distribution of pressure while in stance or gait. The brunt of the weight falls over metatarsal heads and the heel. In patients with DM the biomechanical changes in the soft tissues of the foot lead to deformities and consequently high pressure points. ${ }^{7}$ Abnormally high foot pressures leads to development of foot ulcers. ${ }^{8,9}$ Furthermore as a result of the deformities, the gait is altered. Both the forefoot and hind-foot pressures has been found be increased in diabetic patients with peripheral neuropathy. ${ }^{10}$ In patients at risk of foot ulceration, screening for loss of protective sensation and evaluation of foot pressure is recommended.

Even when the ulcer develops, proper evaluation and regulation of foot pressure may aid in faster healing. Foot pressure can be measured as static pressure i.e. during standing or as dynamic pressure i.e. during gait. Many devices are available to determine the distribution of plantar pressure. They can be in shoe or out of shoe pressure measurement. One of the in shoe pressure measurement system that has been commonly used is the Pedar ${ }^{\circledR}$ system. ${ }^{11}$ Before we endeavour to discuss various offloading techniques, one important aspect of diabetic foot care needs to be considered, i.e. patient education.

\section{Patient education}

\section{Maintain near-normal blood glucose concentration}

Research has shown that maintaining normoglycemia in a diabetic patient is effective in delaying the onset and progress of complications of diabetes such as foot ulcers and neuropathy. ${ }^{12}$ UK Prospective Diabetes Study (UKPDS) Group found that lower median glycated haemoglobin $(\mathrm{HbAlc})$ levels were associated with risk reductions in developing diabetes-related complications. ${ }^{13}$ Thus it is of utmost importance to control the glycaemic levels of a diabetic patient by any means to avoid developing foot ulcers.

\section{Reduction in body weight}

An increase in body weight has been associated with increased risk of developing foot ulcers. This is due to the increased foot pressure in heavier patients. ${ }^{14}$ However, a study by Cavanagh et al., ${ }^{15}$ suggested that it is the structural deformity in diabetic patients that has a larger impact on the development of ulcers, rather than the gain in body weight. Nevertheless, they agreed that an increased body mass contributes in adding on to the total forces on the foot during walking.

\section{Cessation of smoking}

Some studies ${ }^{16,17}$ have found an association of smoking in diabetics with the risk of development of ulcers and amputation, but other studies ${ }^{18}$ have denied the relationship. Nevertheless, it is better to avoid smoking as it has been found that cessation of smoking reduces the risk of vascular disease complications. ${ }^{19}$ Same goes for alcohol 
consumption, even though the evidence to link alcohol consumption and smoking with diabetic foot ulcers is weak.

\section{Self-monitoring}

Patient should adopt self-care practices of maintaining foot hygiene and nail care. Feet should with wash gently with soap and water followed by application of skin moisturizers. Comorbidities like hypertension and hyperlipidemias should be properly treated to as they may predispose to vascular occlusion. Patients should get their feet examined for vibration testing on annual basis. ${ }^{20}$ Patients with DFU should take every care prevents its recurrence.

\section{Offloading}

The main aim of off-loading, or pressure modulation, is to redistribute plantar pressures so that there will be a resultant reduction foot pressures, shock and shear. ${ }^{21}$ The various methods of offloading include bed rest, wheelchair, crutches, total-contact casts, felted foam dressings, half shoes, therapeutic shoes, padded socks, shoe inserts, removable cast walkers. Surgical offloading is usually reserved for chronic deformities or ulcers that are not amenable to conservative means.

\section{Total Contact Cast (TCC)}

TCC is considered the "gold standard" in treating diabetic foot ulcers. Although some places, very few specialists use this "gold standard" for treating majority of diabetic foot ulcers. ${ }^{22}$ TCC is a method where a non-removable cast is fitted around and is in contact with the foot and part of the leg. ${ }^{23}$ The cast is commonly applied to a patient lying in prone position with a flexed knee and the ankles in neutral position to allow for full access to the sole of the foot. ${ }^{24}$ Another advantage of this position is that it allows the gastrosoleus complex to shift proximally, resulting in better fit of the cast. ${ }^{25}$ After applying a thin dressing to the ulcer, foam padding is applied to the bony prominences, Achilles tendon and anterior region of shin to avoid formation of pressure ulcers. Next, an adhesive tape is applied covering the area from the tibial tuberosity to the dorsum of foot followed by a layer of stockinet from the toes to just below the knee. Plaster of Paris rolls are then applied and moulded carefully according to the contours of the foot. Fibre-glass or specialised soles can be used to reinforce the cast with to avoid the pressures that may be caused by weight bearing. ${ }^{26-28}$

A study compared the effectiveness of using TCC compared to traditional dressing treatment and concluded that the use of TCC resulted in significantly more ulcer healing and less infection. ${ }^{29}$ Another study showed that the use of fibre-glass material in TCC reduced the chances of neuropathic plantar ulcers and had a higher patient acceptance compared with using a cloth shoe with rigid soles with unloading alkaform insoles. Also, it recommended the use of fibre-glass bandage of variable rigidity in the treatment of diabetic foot ulcers. ${ }^{30} \mathrm{~A}$ randomised control trial, compared the effectiveness of TCCs, removable cast-walkers (RCWs), and half shoes in the treatment of diabetic foot ulcers and concluded that TCCs heal foot ulcers in a shorter duration compared to the other methods of treatment used. ${ }^{31}$ Furthermore it has been shown that the average cost of treatment of foot ulcer is halved by using TCCs. ${ }^{32}$

The main aim of TCC is to increase the weight-bearing surface area so the pressure is distributed more evenly in the sole of the foot. ${ }^{33}$ Pressure is the amount of force acting perpendicularly per unit area. As the surface area increases with TCC, the force acting perpendicularly reduces, thereby reducing the pressure produced at the prominent parts on the sole of the foot and results in a more evenly distributed pressure. ${ }^{34}$ The reduction and redistribution of pressure is believed to result in the formed ulcer healing quickly and completely. ${ }^{35}$ The decreased local pressure also plays a role in preventing the development of new plantar ulcers. ${ }^{36}$

However, there are factors that hinder the use of the "gold standard" TCCs in the care and management of diabetic foot ulcers in every patient. The application of TCCs is time-consuming ${ }^{22}$ and is often directly proportional with the amount of time spent by a healthcare professional in learning the correct methods of applying the cast. In addition, application of TCCs prevents the daily assessment of foot or wound by the patient or healthcare providers. ${ }^{37}$ Patient may encounter difficulties in conducting activities of daily living, for example bathing, thus reducing compliance. TCCs would also exacerbate postural instability ${ }^{38} \mathrm{TCC}$, if poorly fitted, can cause irritation to the underlying skin and promote the formation of new ulcers, thus becoming counterproductive. ${ }^{22}$ The application of TCCs are contraindicated in acute infection, ischaemia ( $\geq$ grade 3 ulcer) or severe vascular disease (Doppler pressure $<0.4),{ }^{39}$ deep ulcers, draining wounds and wounds in the posterior heel. ${ }^{40}$ In such cases, an alternative treatment for the foot ulcers should be sought.

\section{Charcot Restraint Orthotic Walker (CROW)/ Neuropathic walker}

CROW is a rigid full foot enclosure ankle-foot orthosis where any residual deformity in the device can be accommodated with custom insoles and orthotics. ${ }^{41,42}$ The main aim of CROW is to uniformly distribute the pressure over the foot as a measure to protect the joints and skin, preventing deformity. ${ }^{43}$ It is used as means to protect the foot after removal of a cast used for treatment of a foot ulcer. ${ }^{44}$ The device is internally lined with soft foam, which may aid in the redistribution of pressure and absorbs shock when the patient is walking. ${ }^{45,46}$ The CROW boots are custom made according to each patient's limb. ${ }^{47}$

The mechanism of action of this device is similar with the TCC, i.e. redistribution of pressure is more evenly in the sole of the foot by increasing the surface area which comes in contact with the ground when walking, thus relatively redirecting pressure away from the ulcer site. ${ }^{48}$ The device helps in immobilisation of the affected limb and protects it to allow sufficient time for ulcer to heal. ${ }^{49}$ The CROW is constructed with two shells, anterior and posterior. After applying stockinet to the limb, the posterior shell is slid into the limb, keeping heel in contact with the bottom of the footplate. Then the anterior shell is applied overlapping with the posterior shell followed by fastening the straps on the device. After 10-15 minutes of application the skin should be examined for induration. Presence of significant red marks, bruises or blisters requires the scrutiny by an orthotist. ${ }^{50-52}$ Other modifications to the CROW such as flaring, adding pads and trimming can be done subsequently to accommodate the foot of the patient. ${ }^{53}$

The advantages of CROW include Patients being able to: bear full weight and ambulate, ${ }^{54}$ remove the equipment for inspection of the ulcers, and apply dressings or topical medication. In addition, CROW also effectively controls oedema of the limb. ${ }^{55}$ Furthermore, its usage has good patient satisfaction. ${ }^{55}$ However, patients with CROW have the ability to remove their device and ambulate without protection, thus leading to an increased risk of trauma and development of ulcers. The device is also heavy and is not suitable for use in a frail individual. In addition, there is a likelihood of irritation to the skin and breakdown, requiring a support stocking to be used with this device. ${ }^{56}$ 


\section{Prefabricated walking brace}

These devices, in contrast to the TCCs, are relatively easy to use, cost effective and are removable, allowing access to the wound for certain procedures such as dressing, application of topical medication and bathing, which may improve patient compliance. ${ }^{57}$ They have been shown to reduce forefoot pressure as effective as or more effective than TCCs. ${ }^{58}$ They act in the same principle as TCCs, where redistribution of pressure over the sole is the main objective. However, as with the CROW, there is a potential for patient's non compliance.

\section{Aircast pneumatic walker}

This walker is a type of prefabricated walking brace. It is a removable semi-rigid plastic off-loading device with internal air cells, a rocker sole for improved ambulation and off-loading and a dual-density insole..$^{59}$ It also reduces oedema and shearing forces by virtue of its air cells. ${ }^{60}$ It reduces the peak plantar pressures to an equal or greater degree compared with TCCs in the forefoot, midfoot and hindfoot as well. ${ }^{61}$ This device can be considered as a valid alternative to TCCs in view of its good healing rate and low rate of developing complications. ${ }^{62}$

\section{Bledsoe conformer boots}

This device consists of auto-mould ('memory') foam and dualdensity mid-sole that promotes off-loading and prevents movement that causes breakdown of skin. It also has an aluminium boot shell which is adjustable to accommodate most leg sizes. This conformer comes with an additional pneumatic option, if required. ${ }^{63}$ The components of this device include a bootie and a boot. After applying the stockinet, the patient's foot is slid into the bootie with the foot centred on the white insole. Then, foam wrap is applied over the forefoot and around the leg. The bootie is then positioned in the walking boot. The white innersole fits inside the black midsole. Patient is made to stand upright for half a minute for the initiation of auto-moulding process. While the patient is standing, the plastic covers are removed from the arms, to make the hook material engage with the pile surface. Finally all the straps on the conformer boot are fastened. ${ }^{64,65}$ Unlike TCCs, the Bledsoe conformer boots can be removed for daily dressing, application of topical medications and washing. The auto-mould foam in the innersole with dual-density midsole helps in excellent loading.

The inner soles of the boots are replaceable in case of wear and tear. ${ }^{66}$ A study by Pollo FE et al., ${ }^{66}$ concluded that they performed as well as TCCs in reducing plantar pressures.$^{66}$ Unlike the pneumatic walker, this device is not custom made as it can be moulded to the shape of an individual's foot. ${ }^{67}$ It comes in various sizes and specifically for left and right side for more appropriate fit. However in severe foot deformities, where the deformed foot does not fit into the confines of the conformer boots, this device is ineffective ${ }^{68}$ Being a removable device, there is potential for patient's non-compliance. ${ }^{69}$ Patients may find it heavy and bulky to wear.

\section{Half-shoes}

This shoe is designed with $10^{\circ}$ dorsiflexion with an elevated heel to avoid contact with the ground. A study by Chantelau et al. ${ }^{70}$ found that the usage of half-shoes in the standard treatment of neuropathic forefoot ulcers reduces the overall ulcer healing time compared to using standard treatment only. However, the pressure reduction is consistently less (64-66\% from baseline) than that produced by TCCs (76-85\% from baseline). ${ }^{58}$ This device is however inexpensive and has a high rate of patient compliance due to its convenient usage. ${ }^{56}$
Furthermore as stated by $\mathrm{Wu}$ et al.,22 "patients are usually more tolerant if slight modifications were made to the footwear they are familiar with, compared to introducing them new and complicated devices". The primary mechanism behind this shoe is similar with the other off-loading devices mentioned, that is, to redistribute the pressure on the sole and thereby promote ulcer healing. However, this device may increase postural instability in patients with DFUs. Moreover, it cannot be used in patients with ankle equinus or bilateral ulcers. $^{71}$

The half-shoes usually allow for ankle plantar flexion during pushoff, thus concentrating pressure on the forefoot. Other off-loading devices include felted foam dressings and post-op shoes. Felted foam dressings are multiple-layer foam with a cut-out area for the ulcer, and placed on the ulcer with the help of adhesive and secondary dressings. They are used along with a post operative shoe. The contraindications to its use include skin disorders, frail skin or allergy to a adhesives. ${ }^{72}$ The advantages of post-op shoes include that they are readily available in various sizes and are modifiable.

The disadvantages are lack of biomechanical support and poor potential for healing of forefoot plantar ulcers. ${ }^{72}$ Due to the bulky and heavy nature of TCC and CROW, the use of other devices like below knee walking boot, or controlled ankle motion walker (CAM) has been advocated..$^{73}$ To overcome the compliance issue of removable cast walkers, it has been advised to wrap them with plater or fiberglass and converting them to so called 'iTCC' (instant total contact cast). ${ }^{74}$ Other alternatives to TCC include Ransart boot and Vacuum Stabilisation Boot. Multifaceted approach using interdisciplinary help is required to tackle the foot problems in patients with DM. Due consideration should be given before choosing an appropriate off-loading device. If the ulcer size is not halved after four weeks of treatment, reinvestigate to assess the limb vascular status, any infection of the ulcer or the offloading device, or patient compliance. Surgical offloading is usually reserved for failed conservative treatment especially for Charcot foot with rocker bottom feet or difficult to treat mid foot ulcers.

\section{Acknowledgments}

None.

\section{Conflict of interest}

Author declares that there is no conflict of interest.

\section{References}

1. Pendsey SP. Understanding diabetic foot. Int $J$ Diabetes Dev Cries. 2010;30(2):75-79.

2. Mayfield JA, Reiber GE, Sanders LJ, et al. Preventive foot care in people with diabetes. Diabetes care. 2003;26(Suppl 1):S78-S79.

3. Boulton AJ, Armstrong DG, Albert SF, et al. Task Force of the Foot Care Interest Group of the American Diabetes Association: Comprehensive foot examination and risk assessment. Endocr Pract. 2008;14(5):576-583.

4. Brand PW. Management of the insensitive limb. Phys Ther. 1979;59(1):8-12.

5. Armstrong DG, Athanasiou KA. The edge effect: how and why wounds grow in size and depth. Clin Podiatr Med Surg. 1998;15(1):105-108.

6. Reiber GE, Vileikyte L, Boyko EJ, et al. Causal pathways for incident lower-extremity ulcers in patients with diabetes from two settings. Diabetes Care. 1999;22(1):157-162. 
7. Wrobel JS, Najafi B. Diabetic foot biomechanics and gait dysfunction. $J$ Diabetes Sci Technol. 2010;4(4):833-845.

8. Duckworth T, Boulton AJ, Betts RP, et al. Plantar pressure measurements and the prevention of ulceration in the diabetic foot. $J$ Bone Joint Surg Br. 1985;67(1):79-85.

9. Murray HJ, Young MJ, Hollis S, et al. The association between callus formation, high pressures and neuropathy in diabetic foot ulceration. Diabetes Med. 1996;13(11):979-982.

10. Sarnow MR, Veves A, Giurini JM, et al. In-shoe foot pressure measurements in diabetic patients with at-risk feet and in healthy subjects. Diabetes Care. 1994;17(9):1002-1006.

11. Natali AN, Forestiero A, Carniel EL, et al. Investigation of foot plantar pressure: experimental and numerical analysis. Med Biol Eng Comput 2010;48(12):1167-1174.

12. Diabetes Control and Complications Trial Research Group. The effect of intensive treatment of diabetes on the development and progression of long-term complications in insulin-dependent diabetes mellitus. $N \mathrm{Engl}$ J Med. 1993;329:977-986.

13. Effect of intensive blood-glucose control with metformin on complications in overweight patients with type 2 diabetes (UKPDS 34). UK Prospective Diabetes Study (UKPDS) Group. Lancet. 1998;352(9131):854-865.

14. Hills AP, Hennig EM, McDonald M, et al. Plantar pressure differences between obese and non-obese adults: a biomechanical analysis. Int $J$ Obes Relat Metab Disord. 2001;25(11):1674-1679.

15. Cavanagh PR, Sims DS, Sanders LJ. Body mass is a poor predictor of peak plantar pressure in diabetic men. Diabetes Care. 1991;14(8):750-755.

16. Chaturvedi N, Abbott CA, Whalley A, et al. Risk of diabetes-related amputation in South Asians vs. Europeans in the UK. Diabetes Med. 2002;19(2):99-104.

17. Jbour AS, Jarrah NS, Radaideh AM, et al. Prevalence and predictors of diabetic foot syndrome in type 2 diabetes mellitus in Jordan. Saudi Med J. 2003;24(7):761-764.

18. Boyko EJ, Ahroni JH, Stensel V, et al. A prospective study of risk factors for diabetic foot ulcer. The Seattle Diabetic Foot Study. Diabetes Care. 1999;22(7):1036-1042.

19. Mayfield JA, Reiber GE, Sanders LJ, et al. Preventive foot care in people with diabetes. Diabetes Care. 2003;26(Suppl 1):S78-S79.

20. Caputo GM, Cavanagh PR, Ulbrecht JS, et al. Assessment and management of foot disease in patients with diabetes. $N$ Engl J Med 1994;331:854-860.

21. Mulder G, Armstrong DG, Seaman S. Standard, appropriate, and advanced care and medical-legal considerations. Wounds. 2003;15(4):92-106.

22. Wu SC, Jensen JL, Weber AK, et al. Use of pressure offloading devices in diabetic foot ulcers: do we practice what we preach? Diabetes Care. 2008;31(11):2118-2119.

23. Baker RE. Total contact casting. Journal of the American Podiatric Medical Association. 1995;85(3):172-176.

24. Jimenez AL. Total Contact Casting. 2003.

25. Piaggesi A, Macchiarini S, Rizzo L, et al. An Off-the-Shelf Instan Contact Casting Device for the Management of Diabetic Foot Ulcers A randomized prospective trial versus traditional fiber glass cast. Diabetes care. 2007;30(3):586-590.

26. Van GH, Siney H, Hartmann-Heurtier A, et al. Non removable, Windowed, Fiber glass Cast Boot in the Treatment of Diabetic Plantar Ulcers Efficacy, safety, and compliance. Diabetes care. 2003;26(10):2848-2852.

27. Jensen BJ, Weber A, Jaakola E. A Helpful Primer on Total Contact Casts DPM. 2003;16(8):52-58.
28. Mueller MJ, Diamond JE, Sinacore DR, et al. Total contact casting in treatment of diabetic plantar ulcers. Controlled clinical trial. Diabetes Care. 1989;12(6):384-388.

29. Caravaggi C, Faglia E, De Giglio R, et al. Effectiveness and safety of a non-removable fibreglass off bearing cast versus a therapeutic shoe in the treatment of neuropathic foot ulcers: a randomized study. Diabetes Care. 2000;23(12):1746-1751

30. Armstrong DG, Nguyen HC, Lavery LA, et al. Off-Loading the Diabetic Foot Wound A randomized clinical trial. Diabetes care. 2001;24(6):1019-1022.

31. Fife CE, Carter MJ, Walker D. Why is it so hard to do the right thing in wound care? Wound Repair Regen. 2010;18(2):154-158.

32. Sinacore DR, Mueller MJ, Diamond JE, et al. Diabetic plantar ulcers treated by total contact casting. A clinical report. Phys Ther. 1987;67(10):1543-1549.

33. Shaw JE, Hsi WL, Ulbrecht JS, et al. The mechanism of plantar unloading in total contact casts: implications for design and clinical use. Foot Ankle International. 1997;18(12):809-817.

34. Myerson M, Papa J, Eaton K, et al. The total-contact cast for management of neuropathic plantar ulceration of the foot. J Bone Joint Surg Am. 1992;74(2):261-269.

35. Veves A, Murray HJ, Young MJ, et al. The risk of foot ulceration in diabetic patients with high foot pressure: a prospective study. Diabetologia. 1992;35(7):660-663.

36. Helm PA, Walker SC, Pullium G. Total contact casting in diabetic patients with neuropathic foot ulcerations. Arch Phys Med Rehabil. 1984;65(11):691-693.

37. Lavery LA, Fleishli JG, Laughlin TJ, et al. Is postural instability exacerbated by off-loading devices in high risk diabetics with foot ulcers? Ostomy Wound Manage. 1998;44(1):26-34

38. Sinacore DR. Total contact casting for diabetic neuropathic ulcers Physical therapy. 1996;76(3):296-301.

39. Guyton GP. An analysis of iatrogenic complications from the tota contact cast. Foot Ankle Int. 2005;26(11):903-907.

40. Farber DC, Juliano PJ, Cavanagh PR, et al. Single stage correction with external fixation of the ulcerated foot in individuals with Charco neuroarthropathy. Foot Ankle Int. 2002;23(2):130-134.

41. Sommer TC, Lee TH. Charcot foot: the diagnostic dilemma. Am Fam Physician. 2001;64(9):1591-1598.

42. Madan SS, Pai DR. Charcot neuroarthropathy of the foot and ankle Orthopaedic surgery. 2012;5(2):86-93.

43. Verity S, Sochocki M, Embil JM, et al. Treatment of Charcot foot and ankle with a prefabricated removable walker brace and custom insole. Foot Ankle Surg. 2008;14(1):26-31.

44. Rajbhandari S, Jenkins RD, Davies C, et al. Charcot neuroarthropathy in diabetes mellitus. Diabetologia. 2002;45(8):1085-1096.

45. McCartan BL, Rosenblum BI. Offloading of the Diabetic Foot: Orthotic and Pedorthic Strategies. Clin Podiatr Med Surg. 2014;31(1):71-88.

46. Robinson C, Major MJ, Kuffel C, et al. Orthotic management of the neuropathic foot: An interdisciplinary care perspective. Prosthet Orthot Int. 2015;39(1):73-81

47. Ellen Sobel DP. Pressure Reduction and Off-Loading the Diabetic Foot 2001;97-116.

48. Smith WB, Moore CA. A proposed treatment algorithm for midfoot Charcot arthropathy. Foot Ankle Spec. 2012;5(1):60-64.

49. Sussman C, Strauss MB, Barry DD, et al. Consideration of motor neuropathy for managing the neuropathic foot. JPO: Journal of Prosthetics and Orthotics. 2005;17(2):S28-S31. 
50. Fisher TK, Armstrong DG. Partial calcanectomy in high-risk patients with diabetes: use and utility of a "hurricane" incisional approach. Eplasty. 2010;10:e17.

51. http://capstoneorthopedic.com/services/ankle-foot-orthosis/

52. Mehta JA, Brown C, Sargeant N. Charcot restraint orthotic walker. Foot Ankle Int. 1998;19(9):619-623.

53. Morgan JM, Biehl WC, Wagner FW. Management of neuropathic arthropathy with the Charcot Restraint Orthotic Walker. Clin Orthop Relat Res. 1993;296:58-63.

54. Snyder RJ, Lanier KK. Offloading difficult wounds and conditions in diabetic patient. Ostomy Wound Manage. 2002;48(1):22-35.

55. Beuker BJ, Deursen RW, Price P, et al. Plantar pressure in off-loading devices used in diabetic ulcer treatment. Wound Repair Regen. 2005;13(6):537-542

56. Fleischli JG, Lavery LA, Vela SA, et al. 1997 William J. Stickel Bronze Award. Comparison of strategies for reducing pressure at the site of neuropathic ulcers. J Am Podiatr Med Assoc. 1997;87(10):466-472.

57. Caravaggi C, Sganzaroli A, Fabbi M, et al. Nonwindowed Nonremovable Fiberglass Off-Loading Cast Versus Removable Pneumatic Cast (Aircast XP Diabetic Walker) in the Treatment of Neuropathic Noninfected Plantar Ulcers A randomized prospective trial. Diabetes Care. 2007;30(10):2577-2578.

58. Myerly SM, Stavosky JW. An alternative method for reducing plantar pressures in neuropathic ulcers. Advances in Skin \& Wound Care. 1997;10(1):26-29.

59. Baumhauer JF, Wervey R, McWilliams J, et al. A comparison study of plantar foot pressure in a standardized shoe, total contact cast, and prefabricated pneumatic walking brace. Foot Ankle Int. 1997;18(1):26-33.

60. Armstrong DG, Lavery LA, Wu S, et al. Evaluation of Removable and Irremovable Cast Walkers in the Healing of Diabetic Foot Wounds A randomized controlled trial. Diabetes Care. 2005;28(3):551-554.

61. Moore J. The Practice Management of Off-loading. 2012;119-128.

62. http://www.abledata.com/organizations/bledsoe-brace-systems-medicaltechnology-inc
63. Bledsoe GR, Bledsoe BO. Inventors: Medical Technology, Inc., assignee. Walking boot for diabetic and other patients. US 7418755. 2008.

64. Pollo FE, Brodsky JW, Crenshaw SJ, Kirksey C. Plantar pressures in fiberglass total contact casts vs. a new diabetic walking boot. Foot Ankle Int. 2003;24(1):45-49.

65. Crenshaw SJ, Pollo FE, Brodsky JW. The effect of ankle position on plantar pressure in a short leg walking boot. Foot Ankle Int. 2004;25(2):69-72.

66. Lavery LA. 5 Questions and Answers about Off-Loading. Advances in Skin \& Wound Care. 2003;16(5):231-234.

67. Snyder RJ, Lanier KK. Diabetes: Offloading difficult wounds. Lower Extremity Review. 2009.

68. Chantelau E, Breuer U, Leisch AC, et al. Outpatient treatment of unilateral diabetic foot ulcers with 'half shoes'. Diabetes Med. 1993;10(3):267-270.

69. Bus SA, Valk GD, Van Deursen RW, et al. The effectiveness of footwear and offloading interventions to prevent and heal foot ulcers and reduce plantar pressure in diabetes: a systematic review. Diabetes Metab Res Rev. 2008;24(Suppl 1):S162-S180.

70. Giacalone V. Off-loading the diabetic foot. 2006:253-263.

71. Atkins C. Evidence-based foot care in patients with diabetes. Lower Extremity Review. 2010.

72. Armstrong DG, Short B, Nixon BP, et al. Technique for fabrication of an "instant" total contact cast for treatment of neuropathic diabetic foot ulcers. J Am Podiatr Med Assoc. 2002;92(7):405-408.

73. Dumont IJ, Lepeut MS, Tsirtsikolou DM, et al. A proof-of-concept study of the effectiveness of a removable device for offloading in patients with neuropathic ulceration of the foot: the Ransart boot. Diabetes Med. 2009;26(8):778-782.

74. Cook JJ, Cook EA. Protected weight bearing during treatment of acute charcot neuroarthropathy: a case series. The Foot and Ankle Online Journal. 2011;4(7):1. 